\title{
Sobrevida e Fatores Prognósticos de Pacientes com Sarcoma de Kaposi Atendidos em um Centro de Assistência de Alta Complexidade em Oncologia
}

doi: https://doi.org/10.32635/2176-9745.RBC.2020v66n3.1142

Survival and Prognostic Factors of Kaposi's Sarcoma Patients Attended at a High Complexity Oncology Care Center

Supervivencia y Factores Pronósticos de Pacientes con Sarcoma de Kaposi Tratados en un Centro de Atención de Alta Complejidad en Oncología

Isabele da Rosa Noronha'; Anne Karin da Mota Borges²; Jeniffer Dantas Ferreira3; Gelcio Luiz Quintella Mendes ${ }^{4}$; Isabela da Rosa Noronha5; Rafael Tavares Jomar ${ }^{6}$

RESUMO

Introduçáo: A sobrevida do sarcoma de Kaposi ainda não é bem conhecida porque os poucos estudos que avaliaram-na foram, em maioria, conduzidos com pessoas vivendo com vírus da imunodeficiência humana (HIV). Objetivo: Avaliar a sobrevida e os fatores prognósticos pré-tratamento de pacientes com sarcoma de Kaposi associado ou náo ao HIV. Método: Estudo retrospectivo realizado em uma coorte hospitalar de 81 pacientes diagnosticados com sarcoma de Kaposi entre 2000 e 2014, atendidos em um centro de assistência de alta complexidade em oncologia da cidade do Rio de Janeiro, Brasil. A probabilidade de sobrevida em cinco anos foi estimada por meio do método de Kaplan-Meier. O modelo semiparamétrico de riscos proporcionais de Cox estimou hazard ratios (HR) e respectivos intervalos de 95\% de confiança (IC95\%). Resultados: A sobrevida global em cinco anos foi de 50,9\% (IC95\%: 38,2-62,3). Os fatores associados ao óbito foram idade $\geq 50$ anos (HR: 4,19; IC95\%: 1,5-11,29) e sorologia anti-HIV positiva (HR: 5,82; IC95\%: 1,90-17,85). Conclusáo: A coorte apresentou sobrevida baixa. O prognóstico foi influenciado pela idade $\geq 50$ anos e sorologia anti-HIV positiva, devendo esses fatores serem considerados na avaliação de risco pré-tratamento.

Palavras-chave: Sarcoma de Kaposi; Análise de Sobrevida; Prognóstico; Institutos de Câncer.

\section{ABSTRACT}

Introduction: The survival of Kaposi's sarcoma is still not well known because the few studies that evaluated it were mostly conducted with people living with human immunodeficiency virus (HIV). Objective: To assess survival and pre-treatment prognostic factors in patients with Kaposi's sarcoma associated or not with HIV. Method: Retrospective study conducted in a hospital cohort of 81 patients diagnosed with Kaposi's sarcoma between the years 2000 and 2014 treated at a high complexity care center in oncology in the city of Rio de Janeiro, Brazil. The probability of 5 -year survival was estimated using the Kaplan-Meier method. Hazard ratios (HR) and respective 95\% confidence intervals $(95 \% \mathrm{CI})$ were estimated following Cox's semi-parametric model of proportional hazards. Results: The overall 5-year survival was 50.9\% (95\%CI: 38.2-62.3). The factors associated with death were age $\geq 50$ years (HR: $4.19 ; 95 \% \mathrm{CI}: 1.5-11.29$ ) and positive anti-HIV serology (HR: 5.82; 95\%CI: 1.90-17.85). Conclusion: The cohort had low survival. The prognosis was influenced by age $\geq 50$ years and positive anti-HIV serology, and these factors should be considered in the pre-treatment risk assessment.

Key words: Sarcoma, Kaposi; Survival Analysis; Prognosis; Cancer Care Facilities.
RESUMEN

Introducción: La supervivencia del sarcoma de Kaposi aún no se conoce bien porque los pocos estudios que lo evaluaron se realizaron, en su mayoría, con personas que viven con el virus de inmunodeficiencia humana (VIH). Objetivo: Evaluar la supervivencia y los factores pronósticos previos al tratamiento en pacientes con sarcoma de Kaposi asociado o no con VIH. Método: Estudio retrospectivo realizado en una cohorte hospitalaria de 81 pacientes diagnosticados con sarcoma de Kaposi entre 2000 y 2014 tratados en un centro de atención oncológica de alta complejidad en la ciudad de Río de Janeiro, Brasil. La probabilidad de supervivencia a cinco años se estimó utilizando el método de Kaplan-Meier. El modelo de riesgos proporcionales semiparamétricos de Cox estimó las razones de riesgo (HR) y los respectivos intervalos de confianza del 95\% (IC95\%). Resultados: La tasa de supervivencia general a cinco ańos fue del 50,9\% (IC95\%: 38,262,3 ). Los factores asociados con la muerte fueron edad $\geq 50$ años (HR: 4,19; IC95\%: 1,5-11,29) y serología positiva contra el VIH (HR: 5,82; IC95\%: 1,90-17,85) Conclusión: La cohorte mostró baja supervivencia. El pronóstico estuvo influenciado por la edad $\geq 50$ años y la serología positiva contra el VIH, y estos factores deben considerarse en la evaluación de riesgos previa al tratamiento.

Palabras clave: Sarcoma de Kaposi; Análisis de Supervivencia; Pronóstico; Instituciones Oncológicas.

1Programa de Residência Multiprofissional em Oncologia do Instituto Nacional de Câncer José Alencar Gomes da Silva (INCA). Rio de Janeiro (RJ), Brasil. Orcid iD: https://orcid.org/0000-0002-6884-5144

${ }^{2}$ Coordenação de Gestão de Pessoas do INCA. Rio de Janeiro (RJ), Brasil. Orcid iD: https://orcid.org/0000-0003-1322-3986

${ }^{3}$ Coordenação de Prevenção e Vigilância do INCA. Rio de Janeiro (RJ), Brasil. Orcid iD: https://orcid.org/0000-0002-6724-5614

${ }^{4}$ Coordenação de Assistência do INCA. Rio de Janeiro (RJ), Brasil. Orcid iD: https://orcid.org/0000-0001-9564-0892

${ }^{5}$ Programa de Residência Multiprofissional em Oncologia do INCA. Rio de Janeiro (RJ), Brasil. Orcid iD: https://orcid.org/0000-0001-8079-8566

${ }^{6}$ Coordenação de Assistência do INCA. Rio de Janeiro (RJ), Brasil. Orcid iD: https://orcid.org/0000-0002-4101-7138

Endereço para correspondência: Rafael Tavares Jomar. Praça Cruz Vermelha, 23 - Centro. Rio de Janeiro (RJ), Brasil. CEP 20230-130. E-mail: rafaeljomar@yahoo.com.br 


\section{INTRODUÇÃO}

O sarcoma de Kaposi (SK) é um tumor mesenquimal causado pelo herpes-vírus humano tipo $8^{1}$, que se manifesta como lesão vascular multifocal frequente em sítios mucocutâneos, mas que também pode afetar linfonodos e vísceras ${ }^{2}$. Foi descrito pela primeira vez em 1872, permanecendo como um tumor raro até o surgimento do vírus da imunodeficiência humana (do inglês, human immunodeficiency virus - HIV) no início dos anos $1980^{2}$. O SK pode manifestar-se de quatro formas distintas: clássico, endêmico, iatrogênico e epidêmico ${ }^{3}$. Considerada a forma mais agressiva da doença, o SK epidêmico está associado ao HIV e é mais frequente entre homens que fazem sexo com homens ${ }^{1}$.

Embora a terapia antirretroviral altamente ativa tenha contribuído para a diminuiçâo da incidência e da mortalidade do $\mathrm{SK}^{1}$, foram estimados 41.799 casos novos e 19.902 mortes para o ano 2018 em todo o mundo. Para o mesmo ano na África, estimaram-se 32.446 casos novos e 17.659 mortes por SK, o que corresponde a, respectivamente, $77,6 \%$ e $88,7 \%$ das estimativas de casos novos e de mortes para todo o mundo. O SK é endêmico em diversos países das Regióes Sul e Oriental do continente africano, com destaque para Malawi, Moçambique, Uganda e Zâmbia, onde é a principal causa de morte por câncer ${ }^{4}$.

Em estudo realizado na África do Sul entre os anos 2000 e 2007 com 6.292 pessoas vivendo com HIV, 215 $(3,4 \%)$ tinham SK: os que recebiam terapia antirretroviral altamente ativa apresentaram taxa de mortalidade de 25/100 pessoas-ano e os que náo recebiam, 70/100 pessoas-ano5. Em Uganda, onde o SK é a segunda neoplasia mais incidente na populaçấo geral ${ }^{4}$, um estudo conduzido com 404 indivíduos com SK associado ao HIV evidenciou probabilidade de sobrevida igual a $65,0 \%$ um ano após o diagnóstico e igual a 57,0\% após dois anos ${ }^{6}$.

Para o ano 2018, nos Estados Unidos, estimaram-se 1.329 casos novos de SK e 117 mortes ${ }^{4}$. Um estudo conduzido nesse país com 4.455 casos de SK em homens com menos de 55 anos entre os anos 2000 e $2013^{7}$ mostrou tendência de queda da incidência da doença entre os brancos, mas náo entre os negros, principalmente entre aqueles do Sul dos Estados Unidos, para os quais foi observado aumento da incidência. Além disso, em comparação com os brancos, os negros apresentaram maior probabilidade de morrer por SK, revelando, assim, a existência de disparidades geográficas e raciais na incidência e na sobrevida do SK nesse país ${ }^{7}$.

No Brasil, entre os anos 1996 e 2010, a incidência de SK era 2,5 vezes maior do que a dos Estados Unidos, e, mesmo nos dias atuais, ainda é a neoplasia mais frequente entre pessoas vivendo com HIV no país 8 . Para o ano 2018, estimaram-se 778 casos novos de SK e 137 mortes por esta causa no Brasil ${ }^{4}$. Um estudo realizado com 3.557 casos de síndrome da imunodeficiência adquirida (aids) em centro de referência da cidade de Sáo Paulo, Brasil, estimou prevalência de SK igual a 6,0\% ${ }^{1}$.

A extensa revisão de literatura efetuada pelos autores revelou que a sobrevida do SK ainda não é bem conhecida, uma vez que os poucos estudos que avaliaram-na foram, em maioria, conduzidos com pessoas vivendo com HIV. A fim de colaborar com o preenchimento dessa lacuna de pesquisa e contribuir com o avanço do conhecimento sobre o tema, o objetivo deste estudo foi avaliar a sobrevida e os fatores prognósticos pré-tratamento de pacientes com SK atendidos em um centro de assistência de alta complexidade em oncologia, onde são tratados pacientes com SK a despeito da situação sorológica para o HIV.

\section{MÉTODO}

Trata-se de um estudo retrospectivo, desenvolvido em uma coorte hospitalar composta por todos os pacientes diagnosticados com SK entre $1^{\circ}$ de janeiro de 2000 e 30 de junho de 2014 e atendidos em um centro de assistência de alta complexidade em oncologia, o Instituto Nacional de Câncer José Alencar Gomes da Silva (INCA), localizado na cidade do Rio de Janeiro, Brasil $(\mathrm{N}=81)$.

A fonte de informação do estudo foi o banco de dados do Registro Hospitalar de Câncer. O prontuário do paciente também foi consultado para buscar informações não coletadas sistematicamente por esse Registro, quais sejam: sorologia anti-HIV, contagem de células CD4, carga viral do HIV e uso de terapia antirretroviral altamente ativa, que, por sua vez, foram registradas em uma ficha de coleta de dados e, na sequência, incluídas no banco de dados fornecido pelo Registro Hospitalar de Câncer.

O início do seguimento de cada paciente correspondeu à data do diagnóstico histopatológico do SK. Para realizar o seguimento de cinco anos, foram adotados os seguintes procedimentos em julho de 2019: consulta ao banco de dados fornecido pelo Registro Hospitalar de Câncer, consulta aos prontuários, consulta ao Sistema de Informaçôes sobre Mortalidade do Estado do Rio de Janeiro e consulta ao Portal Extrajudicial de Consulta de Nascimentos e Óbitos do Poder Judiciário do Estado do Rio de Janeiro.

As variáveis investigadas no momento do diagnóstico do SK foram: sexo (masculino ou feminino), idade (contínua e categorizada conforme a mediana em $<50$ anos e $\geq 50$ anos), raça/cor da pele (categorizada em branca e preta e/ou parda), escolaridade (categorizada em nenhuma, 
ensino fundamental incompleto e/ou completo e ensino médio e/ou superior), situaçáo conjugal (categorizada em vive com companheiro(a) e vive sem companheiro(a)), sorologia anti-HIV (positiva ou negativa), contagem de células CD4 (categorizada em $<200$ células $/ \mathrm{mm}^{3}$ e $\geq 200$ células $/ \mathrm{mm}^{3}$ ), carga viral do HIV indetectável (sim ou não) e uso de terapia antirretroviral altamente ativa (sim ou não).

Inicialmente, foi realizada uma análise exploratória dos dados por meio da descrição da distribuição das variáveis na população de estudo. Para a variável contínua idade, foram calculadas a mediana, a média e seu respectivo desvio-padrão (DP). Para as demais variáveis categóricas, foram calculadas proporçôes, sendo a comparaçâo entre os grupos realizada pelo teste exato de Fisher ou qui-quadrado de Pearson. Em ambos os testes, foi considerado estatisticamente significativo $p<0,05$.

Para estimar a probabilidade de sobrevida em cinco anos e seu respectivo intervalo de 95\% de confiança (IC95\%), foi aplicado o método de Kaplan-Meier com os seguintes critérios: i) evento inicial: diagnóstico histopatológico do SK; ii) evento final: óbito, independente da causa; iii) tempo de sobrevida: tempo entre os eventos inicial e final; e iv) censuras: casos perdidos durante o seguimento e casos vivos ao fim desse período. Em seguida, foram estimadas funçôes de sobrevida - global e segundo as variáveis do estudo - e seus respectivos IC95\%. Para comparar as curvas de sobrevida, foi utilizado o teste de log-rank, considerando-se $p<0,05$.

Para avaliar os efeitos dos fatores prognósticos sobre o tempo de sobrevida da coorte, foram estimadas hazard ratios (HR) e seus respectivos IC95\%, seguindo-se o modelo semiparamétrico de riscos proporcionais de Cox. Todas as variáveis do estudo foram incluídas conjuntamente na análise multivariada, pois não violaram o pressuposto dos riscos proporcionais, avaliado por meio do teste de suposição de riscos proporcionais, considerando-se $p<0,05$, no software Stata versão 15.0, no qual todas as análises foram realizadas.

$\mathrm{Na}$ condução do estudo, foram integralmente observadas as normas constantes nas Resoluçóes do Conselho Nacional de Saúde n. ${ }^{\circ}$ 466, de 12 de dezembro de 2012, e n. ${ }^{\circ}$ 510, de 7 de abril de 2016. Assim, ele foi aprovado pelo Comitê de Ética em Pesquisa do INCA em 25 de maio de 2019 sob o número de parecer 3.347.762 e CAAE: 12620919.2.0000.5274.

\section{RESULTADOS}

A Tabela 1 apresenta as características sociodemográficas e clínicas da população de estudo. Os homens corresponderam a $71,6 \%$ dos casos de SK. A mediana da idade ao diagnóstico foi de 50 anos e a média igual a 52,6 anos $(\mathrm{DP}=18,7)$. Além disso, 62,2\% da coorte tinha sorologia anti-HIV positiva.

Tabela 1. Características sociodemográficas e clínicas dos casos de sarcoma de Kaposi atendidos em um centro de assistência de alta complexidade em oncologia. Rio de Janeiro, 2000-2014 ( $\mathrm{N}=81$ )

\begin{tabular}{|c|c|c|}
\hline Variáveis & $\mathbf{N}$ & $\%$ \\
\hline \multicolumn{3}{|l|}{ Sexo } \\
\hline Masculino & 58 & 71,6 \\
\hline Feminino & 23 & 28,4 \\
\hline Total & 81 & 100 \\
\hline \multicolumn{3}{|l|}{ Idade } \\
\hline$<50$ anos & 37 & 45,7 \\
\hline$\geq 50$ anos & 44 & 54,3 \\
\hline Total & 81 & 100 \\
\hline \multicolumn{3}{|l|}{ Raça/cor da pele ${ }^{a}$} \\
\hline Branca & 37 & 46,2 \\
\hline Preta e/ou parda & 43 & 53,8 \\
\hline Total & 80 & 100 \\
\hline \multicolumn{3}{|l|}{ Escolaridade $^{a}$} \\
\hline Nenhuma & 05 & 6,3 \\
\hline $\begin{array}{l}\text { Ensino fundamental incompleto e/ou } \\
\text { completo }\end{array}$ & 39 & 49,4 \\
\hline Ensino médio e/ou superior & 35 & 43,3 \\
\hline Total & 79 & 100 \\
\hline \multicolumn{3}{|l|}{ Situação conjugala } \\
\hline Vive sem companheiro(a) & 47 & 59,5 \\
\hline Vive com companheiro(a) & 32 & 40,5 \\
\hline Total & 79 & 100 \\
\hline \multicolumn{3}{|l|}{ Sorologia anti-HIV ${ }^{a}$} \\
\hline Negativa & 28 & 37,8 \\
\hline Positiva & 46 & 62,2 \\
\hline Total & 74 & 100 \\
\hline
\end{tabular}

Legendas: ${ }^{\mathrm{M}}$ Missings: raça/cor da pele $(\mathrm{N}=1 ; 1,2 \%)$; escolaridade $(\mathrm{N}=2 ; 2,5 \%)$; situação conjugal $(\mathrm{N}=2 ; 2,5 \%)$; sorologia anti-HIV $(\mathrm{N}=7 ; 8,6 \%)$.

Ao analisar a distribuição das características sociodemográficas da coorte segundo o status da sorologia anti-HIV, os positivos apresentaram maior proporção de casos masculinos $(82,6 \%)$ comparados aos negativos $(54,1 \%)$. Em relação à faixa etária, $73,9 \%$ dos positivos tinham idade $<50$ anos frente a $7,1 \%$ dos negativos. No que diz respeito à escolaridade, $54,5 \%$ dos positivos tinham nível médio e/ou superior comparados a 28,6\% dos negativos (Tabela 2).

Considerando apenas aqueles com sorologia anti-HIV positiva no momento do diagnóstico de SK, 15,4\% 
apresentavam carga viral do HIV indetectável, 60,0\% tinham contagem de células CD $4<200$ células $/ \mathrm{mm}^{3}$ e $10,5 \%$ não faziam uso de terapia antirretroviral altamente ativa (Tabela 2). No entanto, a ausência de informação para essas variáveis é da ordem de $71,7 \%, 56,5 \%$ e $58,7 \%$, respectivamente.
Foram observados durante os cinco anos de seguimento $49(60,5 \%)$ censuras e $32(39,5 \%)$ óbitos. O grupo censurado foi constituído por $29(59,2 \%)$ pacientes vivos ao fim dos cinco anos de seguimento e 20 (40,8\%) perdidos no período. Os casos perdidos tinham média de idade ao diagnóstico de 45,15 anos (DP=18,55) e

Tabela 2. Características sociodemográficas e clínicas dos casos de sarcoma de Kaposi atendidos em um centro de assistência de alta complexidade em oncologia segundo status da sorologia anti-HIV. Rio de Janeiro, 2000-2014 (N=81)

\begin{tabular}{|c|c|c|c|}
\hline \multirow[b]{2}{*}{ Variáveis } & \multicolumn{2}{|c|}{ Sorologia anti-HIV } & \multirow[b]{2}{*}{$p^{a}$} \\
\hline & $\begin{array}{c}\text { Negativa } \\
\mathbf{N}(\%)\end{array}$ & $\begin{array}{c}\text { Positiva } \\
\text { N (\%) }\end{array}$ & \\
\hline \multicolumn{4}{|l|}{ Sexo } \\
\hline Masculino & $16(57,1)$ & $38(82,6)$ & \multirow{3}{*}{0,02} \\
\hline Feminino & $12(42,9)$ & $08(17,4)$ & \\
\hline Total & $28(100)$ & $46(100)$ & \\
\hline \multicolumn{4}{|l|}{ Idade } \\
\hline$<50$ anos & $02(7,1)$ & $34(73,9)$ & \multirow{3}{*}{$<0,01$} \\
\hline$\geq 50$ anos & $26(92,9)$ & $12(26,1)$ & \\
\hline Total & $28(100)$ & $46(100)$ & \\
\hline \multicolumn{4}{|l|}{ Raça/cor da pele } \\
\hline Branca & $15(53,6)$ & $16(35,6)$ & \multirow{3}{*}{0,15} \\
\hline Preta e/ou parda & $13(46,4)$ & $29(64,4)$ & \\
\hline Total & $28(100)$ & $45(100)$ & \\
\hline \multicolumn{4}{|l|}{ Escolaridade $^{b}$} \\
\hline Nenhuma & $04(14,3)$ & $01(2,3)$ & \multirow{4}{*}{0,03} \\
\hline Ensino fundamental incompleto e/ou completo & $16(57,1)$ & $19(43,2)$ & \\
\hline Ensino médio e/ou superior & $08(28,6)$ & $24(54,5)$ & \\
\hline Total & $28(10)$ & $44(100)$ & \\
\hline \multicolumn{4}{|l|}{ Situação conjugalb } \\
\hline Vive sem companheiro(a) & $13(46,4)$ & $31(68,9)$ & \multirow{3}{*}{0,08} \\
\hline Vive com companheiro(a) & $15(53,6)$ & $14(31,1)$ & \\
\hline Total & $28(100)$ & $45(100)$ & \\
\hline \multicolumn{4}{|l|}{ Contagem de células CD4 ${ }^{\text {b }}$} \\
\hline$<200$ células $/ \mathrm{mm}^{3}$ & - & $12(60,0)$ & \multirow{3}{*}{ - } \\
\hline$\geq 200$ células $/ \mathrm{mm}^{3}$ & - & $08(40,0)$ & \\
\hline Total & - & $20(100)$ & \\
\hline \multicolumn{4}{|l|}{ Carga viral do HIV indetectável ${ }^{b}$} \\
\hline Sim & - & $02(15,4)$ & \multirow{3}{*}{-} \\
\hline Não & - & $11(84,6)$ & \\
\hline Total & - & $13(100)$ & \\
\hline \multicolumn{4}{|l|}{ Terapia antirretroviral altamente ativa ${ }^{b}$} \\
\hline Sim & - & $17(89,5)$ & \multirow{3}{*}{-} \\
\hline Não & - & $02(10,5)$ & \\
\hline Total & - & 19 (100) & \\
\hline
\end{tabular}

Legendas: ${ }^{\mathrm{a}} \mathrm{p}$-valor correspondente aos testes exato de Fisher ou qui-quadrado de Pearson; ${ }^{\mathrm{b}}$ Missings: raça/cor da pele $(\mathrm{N}=1 ; 1,4 \%) ;$ escolaridade $(\mathrm{N}=2 ; 2,7 \%) ;$ situaçăo conjugal $(\mathrm{N}=1 ; 1,4 \%)$; contagem de células CD4 ( $=26 ; 56,5 \%)$; carga viral do HIV indetectável $(\mathrm{N}=33 ; 71,7 \%)$; terapia antirretroviral altamente ativa $(\mathrm{N}=27 ; 58,7 \%)$. 
tempo médio de seguimento de 22,7 meses ( $\mathrm{DP}=15,81$ ). Quando comparado aos demais casos da coorte, o grupo de pacientes perdidos durante o seguimento apresentou maior proporção de casos do sexo masculino $(90,0 \%$; $p=0,04)$ e com idade $<50$ anos $(70,0 \% ; p=0,02)$.

O tempo médio de seguimento da coorte foi de 33,5 $(\mathrm{DP}=23,1)$ meses, sendo de 44,1 ( $\mathrm{DP}=21,2)$ para os casos censurados e de 18,9 (DP=17,1) para os casos de óbito.

Dos 32 óbitos observados, $61,7 \%$ eram do sexo masculino, $70,6 \%$ tinham idade $\geq 50$ anos, $52,9 \%$ eram brancos, $44,1 \%$ possuíam ensino médio e/ou superior, $54,5 \%$ viviam sem companheiro(a) e $65,5 \%$ apresentavam sorologia anti-HIV positiva.

A probabilidade de sobrevida global da coorte em cinco anos foi de 50,9\% (IC95\%: 38,2-62,3) (Figura 1). A Tabela 3 apresenta a probabilidade condicional de sobrevida em cinco anos segundo as variáveis sociodemográficas e clínicas da população de estudo. Não foram observadas diferenças significativas entre os grupos em nenhuma das curvas de sobrevida estimadas.

Tabela 3. Probabilidade condicional de sobrevida em cinco anos segundo as características sociodemográficas e clínicas para a coorte de pacientes com sarcoma de Kaposi. Rio de Janeiro, 2000-2014

\begin{tabular}{|c|c|c|}
\hline Variáveis & $\begin{array}{l}\text { Probabilidade } \\
\text { de sobrevida } \\
\text { (IC95\%) }\end{array}$ & $p^{b}$ \\
\hline Sexo & & \multirow{3}{*}{0,20} \\
\hline Masculino & $50,9(38,2-62,3)$ & \\
\hline Feminino & $40,8(20,4-60,3)$ & \\
\hline Idade & & \multirow{3}{*}{0,06} \\
\hline$<50$ anos & $66,6(46,2-80,7)$ & \\
\hline$\geq 50$ anos & $39,8(24,4-54,8)$ & \\
\hline Raça/cor da pele & & \multirow{3}{*}{0,17} \\
\hline Branca & $37,1(18,7-55,6)$ & \\
\hline Preta e/ou parda & $60,2(43,2-73,5)$ & \\
\hline Escolaridade & & \multirow{4}{*}{0,40} \\
\hline Nenhuma & $20,0(8,00-58,1)$ & \\
\hline $\begin{array}{l}\text { Ensino fundamental } \\
\text { incompleto e/ou } \\
\text { completo }\end{array}$ & $55,2(36,3-70,5)$ & \\
\hline $\begin{array}{l}\text { Ensino médio e/ou } \\
\text { superior }\end{array}$ & $49,6(29,9-66,6)$ & \\
\hline Situação conjugal & & \multirow{3}{*}{0,65} \\
\hline $\begin{array}{l}\text { Vive sem } \\
\text { companheiro(a) }\end{array}$ & $52,7(35,2-67,6)$ & \\
\hline $\begin{array}{l}\text { Vive com } \\
\text { companheiro(a) }\end{array}$ & $48,4(29,1-65,3)$ & \\
\hline Sorologia anti-HIV & & \multirow{3}{*}{0,51} \\
\hline Negativa & $61,9(40,5-77,5)$ & \\
\hline Positiva & $50,0(32,7-65,0)$ & \\
\hline
\end{tabular}

Legendas: ${ }^{\mathrm{a} I C} 95 \%$ : intervalo de $95 \%$ de confiança; ${ }^{\mathrm{b}} p$-valor correspondente ao teste de log-rank.

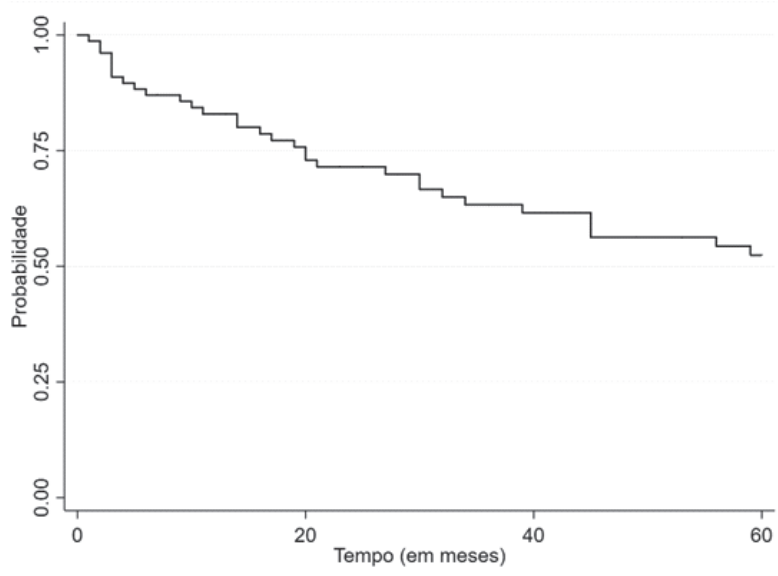

Figura 1. Curva de Kaplan-Meier exibindo a sobrevida em cinco anos para a coorte de pacientes com sarcoma de Kaposi. Rio de Janeiro, 2000-2014

A Tabela 4 apresenta as HR brutas e ajustadas estimadas segundo os modelos de riscos proporcionais de Cox. O modelo multivariado mostrou que, independentemente das demais variáveis, idade $\geq 50$ anos (HR=4,19; IC95\%: $1,55-11,29)$ e sorologia anti-HIV positiva $(\mathrm{HR}=5,82$; IC95\%: 1,90-17,85) apresentaram-se como fatores prognósticos para o óbito em cinco anos.

\section{DISCUSSÃO}

O objetivo deste estudo foi avaliar a sobrevida e os fatores prognósticos pré-tratamento de pacientes com SK atendidos em um centro de assistência de alta complexidade em oncologia brasileiro. Seus resultados mostram que, ao fim de cinco anos de seguimento, a sobrevida global foi de $50,9 \%$ e a idade $\geq 50$ anos e a sorologia anti-HIV positiva apresentaram-se como fatores independentes de pior prognóstico.

Na literatura disponível, observa-se que a maioria dos estudos, que avaliaram a sobrevida do SK, era de pessoas vivendo com HIV atendidas em centros de referência em infectologia, o que dificultou a comparação de nossos resultados, pois $37,8 \%$ dos pacientes da coorte tinham sorologia anti-HIV negativa. Cabe destacar, entretanto, que o SK é uma neoplasia definidora da aids ${ }^{1}$, reconhecida como a primeira doença oportunista associada ao $\mathrm{HIV}^{9} \mathrm{e}$ como o tumor mais prevalente nessa população ${ }^{10}$.

Diante disso, nos limites da comparação, os resultados do presente estudo evidenciam que a sobrevida do SK em cinco anos é inferior à observada em estudos realizados na Alemanha $(96,8 \%)^{11}$, Inglaterra $(89,0 \%)^{12}$ e Itália $(79,1 \%)^{13}$ entre pessoas vivendo com HIV em uso de terapia antirretroviral altamente ativa. Acredita-se que tal diferença seja devida ao estudo ter sido realizado em um centro de assistência de alta complexidade em 
Tabela 4. Hazard ratios brutas e ajustadas para a coorte de pacientes com sarcoma de Kaposi. Rio de Janeiro, 2000-2014

\begin{tabular}{|c|c|c|c|}
\hline Variáveis & $\begin{array}{l}\text { HR bruta } \\
(\text { IC95\%)a }\end{array}$ & $\mathbf{p}^{\mathbf{b}}$ & $\begin{array}{l}\text { HR ajustadac } \\
(1 \mathrm{C} 95 \%)^{a}\end{array}$ \\
\hline \multicolumn{4}{|l|}{ Sexo } \\
\hline Masculino & 1,00 & \multirow{2}{*}{0,83} & 1,00 \\
\hline Feminino & $1,56(0,77-3,17)$ & & $1,73(0,56-5,30)$ \\
\hline \multicolumn{4}{|l|}{ Idade } \\
\hline$<50$ anos & 1,00 & \multirow{2}{*}{0,58} & 1,00 \\
\hline$\geq 50$ anos & $1,99(0,94-4,21)$ & & $4,19(1,55-11,29)$ \\
\hline \multicolumn{4}{|l|}{ Raça/cor da pele } \\
\hline Branca & 1,00 & \multirow{2}{*}{0,34} & 1,00 \\
\hline Preta e/ou parda & $0,61(0,30-1,24)$ & & $0,55(0,21-1,39)$ \\
\hline \multicolumn{4}{|l|}{ Escolaridade } \\
\hline Nenhuma & 1,00 & \multirow{3}{*}{0,53} & 1,00 \\
\hline Ensino fundamental incompleto e/ou completo & $0,47(0,15-1,44)$ & & $0,53(0,12-2,22)$ \\
\hline Ensino médio e/ou superior & $0,55(0,18-1,69)$ & & $0,58(0,11-3,00)$ \\
\hline \multicolumn{4}{|l|}{ Situação conjugal } \\
\hline Vive sem companheiro(a) & $0,85(0,42-1,73)$ & \multirow{2}{*}{0,68} & $0,66(0,28-1,54)$ \\
\hline Vive com companheiro(a) & 1,00 & & 1,00 \\
\hline \multicolumn{4}{|l|}{ Sorologia anti-HIV } \\
\hline Negativa & 1,00 & \multirow{2}{*}{0,87} & 1,00 \\
\hline Positiva & $1,29(0,59-2,82)$ & & $5,82(1,90-17,85)$ \\
\hline
\end{tabular}

Legendas: aC95\%: intervalo de 95\% de confiança; ${ }^{b}$-valor correspondente ao teste de proporcionalidade dos riscos; ${ }^{\mathrm{A}} \mathrm{HR}$ : hazard ratios ajustada por todas as variáveis.

oncologia de referência no Brasil para onde costumam ser encaminhados casos de SK associado ao HIV, em que a terapia antirretroviral altamente ativa náo obteve sucesso no tratamento da neoplasia.

Com base em dados do Surveillance, Epidemiology, and End Results, fonte oficial de informaçóes para vigilância do câncer nos Estados Unidos, a sobrevida relativa em cinco anos de indivíduos diagnosticados com SK em 2011 no país foi de $77,6 \%{ }^{14}$. Um estudo brasileiro realizado com uma coorte hospitalar de 535 pacientes acometidos por diversos tipos de câncer entre os anos 2005 e 2012 relatou sobrevida do SK em cinco anos de aproximadamente $65,0 \%{ }^{15}$. Cabe ressaltar que todos os pacientes com diagnóstico de SK no referido estudo tinham sorologia anti-HIV positiva.

Em comparação com a sobrevida descrita por essas pesquisas, a do presente estudo também é inferior. Uma explicação razoável para esse achado é que o grande número de censuras por motivo de perda durante o seguimento ativo pode ter subestimado a sobrevida, pois é provável que a maioria desses casos censurados esteja viva, caso contrário, o óbito teria sido identificado nas fontes oficiais de informaçáo consultadas ${ }^{16}$.
Embora o presente estudo não tenha verificado diferenças nas curvas de sobrevida segundo raça/cor da pele, nos Estados Unidos, identificou-se a existência de disparidades raciais na sobrevivência por $S K$, uma vez que, entre os 4.455 casos de SK em homens com menos de 55 anos diagnosticados de 2000 a 2013, a sobrevida específica em cinco anos entre os afro-americanos $(63,3 \%)$ foi significativamente menor $(p<0,01)$ do que a dos brancos $(75,5 \%)$ e do que a de outras raças/etnias $(74,0 \%)^{7}$.

Vale ressaltar, entretanto, que, a despeito de significância estatística, o estudo mostra que pretos e/ou pardos têm maior sobrevida e menor risco de óbito em comparação aos brancos. Esse resultado pode ser em razão da categorização da variável estabelecida em função da pequena amostra do estudo, posto que aqueles de raça/cor da pele preta ou parda foram reunidos em única categoria. Assim, é possível que os pardos estejam mascarando a sobrevida e o risco de óbito dos pretos. Diante disso, estudos futuros e com amostras maiores do que a deste estudo são necessários para investigar diferenças na sobrevida em relação à raça/ cor da pele e suas possíveis causas.

Em investigações realizadas em centros de referência em infectologia junto a pessoas vivendo com HIV, alguns fatores prognósticos de pior sobrevida já foram descritos, 
quais sejam: estadiamento avançado do tumor ${ }^{5,6,17,18}$, doenças sistêmicas concomitantes ${ }^{5,6,17}$, comprometimento pulmonar e hepático ${ }^{19}$, diagnóstico tardio do $\mathrm{HIV}^{5}$, contagem de células CD4 baixa $\left(<200 \text { células } / \mathrm{mm}^{3}\right)^{18,19}$ e detecção sorológica do herpes-vírus humano tipo 8 ao diagnóstico ${ }^{18}$. Nessa investigação, idade $\geq 50$ anos e sorologia anti-HIV positiva foram os únicos fatores independentes de pior prognóstico identificados.

De modo controverso, outras pesquisas não identificaram a idade como um preditor da sobrevida do SK ${ }^{5,6,17-19}$. Os resultados deste estudo são diferentes: diante do controle por diversas variáveis de confusão, inclusive sorologia anti-HIV, o efeito da idade $\geq 50$ anos sobre o óbito foi evidenciado. Além disso, na comparação das curvas de sobrevida estimadas de acordo com a idade, a significância estatística mostrou-se limítrofe - possivelmente por causa do pequeno tamanho da amostra do estudo - o que parece corroborar a influência dessa variável na sobrevida do SK. Por outro lado, acredita-se que o efeito da idade avançada sobre o óbito também se deve ao fato de esta pesquisa ter sido conduzida não somente com casos de SK associado ao HIV, mas também com outros tipos de SK (infelizmente, não classificados em prontuário), tal como o clássico que acomete preferencialmente pessoas com idade entre 40 e $70 \operatorname{anos}^{20}$.

Entre as limitaçôes do estudo, merece destaque o grande número de censuras por motivo de perda durante o seguimento ativo que, além da tendência para subestimar a probabilidade de sobrevida, pode ter produzido um viés decorrente das perdas diferenciais de seguimento ${ }^{16}$. Ademais, a sobrevida avaliada não foi doença-específica, já que não foi possível identificar a causa do óbito de todos os pacientes. O pequeno número de participantes na coorte, embora composta por todos os pacientes com SK atendidos pelo cenário de estudo no período investigado, não permitiu estimativas precisas de HR que apresentaram largos IC95\%. Por fim, a ausência de registro em prontuário do estadiamento do tumor e os poucos registros de contagem de células CD4, carga viral do HIV e uso de terapia antirretroviral altamente ativa entre aqueles com sorologia anti-HIV positiva inviabilizaram a provável identificação dessas variáveis como fatores prognósticos.

Não obstante suas limitaçóes, cabe destacar a originalidade do estudo, uma vez que a sobrevida do SK foi avaliada em um centro de assistência de alta complexidade em oncologia, onde são tratados pacientes com SK independentemente da situação sorológica para o HIV. Assim, acredita-se que sejam necessários novos estudos, notadamente multicêntricos, em virtude da raridade da neoplasia, a fim de se obter um panorama da sobrevida e dos fatores prognósticos de pacientes com SK não associado ao HIV.

\section{CONCLUSÃO}

Ao fim de cinco anos de seguimento, a sobrevida global da coorte foi de 50,9\%. A idade $\geq 50$ anos e a sorologia anti-HIV positiva apresentaram-se como fatores prognósticos independentes para o óbito de pacientes com SK atendidos em um centro de assistência de alta complexidade em oncologia. Logo, por causa de seu grande poder preditivo, idade e sorologia anti-HIV devem ser utilizadas na avaliação de risco pré-tratamento de pacientes com SK.

\section{CONTRIBUIÇÕES}

Isabele da Rosa Noronha e Anne Karin da Mota Borges contribuíram na análise de dados, interpretação dos resultados e na redação do manuscrito. Jeniffer Dantas Ferreira, Gelcio Luiz Quintella Mendes e Isabela da Rosa Noronha contribuíram na revisão crítica e na redação. Rafael Tavares Jomar contribuiu na concepçáo e delineamento do estudo, análise de dados, interpretação dos resultados e redação do manuscrito. Todos os autores aprovaram a versão final a ser publicada.

\section{DECLARAÇÃO DE CONFLITO DE INTERESSES}

Nada a declarar.

\section{FONTES DE FINANCIAMENTO}

Não há.

\section{REFERÊNCIAS}

1. Tancredi MV, Pinto VM, Silva MH, et al. Prevalência de sarcoma de Kaposi em pacientes com aids e fatores associados, São Paulo-SP, 2003-2010. Epidemiol Serv Saúde. 2017;26(2):379-87. doi: http://doi.org/10.5123/ s1679-49742017000200015

2. Schneider JW, Dittmer DP. Diagnosis and treatment of Kaposi sarcoma. Am J Clin Dermatol. 2017;18(4):52939. doi: http://doi.org/10.1007/s40257-017-0270-4

3. Koenigkam CAR, Frota MA, Daiha E, et al. Sarcoma de Kaposi simulando granuloma piogênico. RBM Rev Bras Med [Internet]. 2014 [acesso 2020 maio 28];71(n. esp. g1):29-32. Disponível em: https://www.researchgate. net/publication/267923469_Sarcoma_de_Kaposi_ simulando_granuloma_piogenico_Kaposis_sarcoma_ simulating_pyogenic_granuloma\#fullTextFileContent

4. Ferlay J, Ervik M, Lam F, et al. Cancer Tomorrow [Internet]. Lyon (FR): International Agency for Research on Cancer; 2018 [cited 2020 May 28]. Available from: https://gco.iarc.fr/tomorrow/home 
5. Chu KM, Mahlangeni G, Swannet S, et al. AIDSassociated Kaposi's sarcoma is linked to advanced disease and high mortality in a primary care HIV programme in South Africa. J Int AIDS Soc. 2010;13:23. doi: http:// doi.org/10.1186/1758-2652-13-23

6. Okuku F, Krantz EM, Kafeero J, et al. Evaluation of a predictive staging model for HIV-associated Kaposi sarcoma in Uganda. J Acquir Immune Defic Syndr. 2017;74(5):548-54. doi: http://doi.org/10.1097/ QAI.0000000000001286

7. Royse KE, Chaer FE, Amirian ES, et al. Disparities in Kaposi sarcoma incidence and survival in the United States: 2000-2013. PLoS One. 2017;12(8):e0182750. doi: http://doi.org/10.1371/journal.pone.0182750

8. Castilho JL, Luz PM, Shepherd BE, et al. HIV and cancer: a comparative retrospective study of Brazilian and U.S. clinical cohorts. Infect Agent Cancer. 2015;10:4. doi: http://doi.org/10.1186/1750-9378-10-4

9. Feller L, Lemmer J. Insights into pathogenic events of HIV-associated Kaposi sarcoma and immune reconstitution syndrome related Kaposi sarcoma. Infect Agent Cancer. 2008;3:1. doi: http://doi. org/10.1186/1750-9378-3-1

10. Robey RC, Bower M. Facing up to the ongoing challenge of Kaposi's sarcoma. Curr Opin Infect Dis. 2015;28(1):31-40. doi: http://doi.org/10.1097/ QCO.0000000000000122

11. Klingenberg RE, Esser S, Brockmeyer NH, et al. Profil der Kaposi-Sarkom-Patienten im Kompetenznetz HIV/ AIDS. Der Hautarzt. 2018;69:143-8. doi: http://doi. org/10.1007/s00105-017-4062-9

12. Bower M, Dalla Pria A, Coyle C, et al. Prospective stagestratified approach to AIDS-related Kaposi's sarcoma. J Clin Oncol. 2014;32(5):409-14. doi: http://doi. org/10.1200/JCO.2013.51.6757

13. Gotti D, Raffetti E, Albini L, et al. Survival in HIVinfected patients after a cancer diagnosis in the cART Era: results of an Italian multicenter study. PLoS One. 2014;9(4):e94768. doi: http://doi.org/10.1371/journal. pone. 0094768

14. National Cancer Institute. Surveillance, Epidemiology, and End Results Program [Internet]. Bethesda: National Cancer Institute; 2020 [cited 2020 May 28]. Available from: https://seer.cancer.gov/csr/1975_2016/browse_csr. php?sectionSEL=10\&pageSEL=sect_10_table.07

15. Nascimento FG. Avaliação da sobrevida em pacientes com neoplasias no período de 2005 a 2012 em um hospital universitário em Salvador, Bahia [monografia]. Salvador (BA): Universidade Federal da Bahia; 2014. [acesso 2020 maio 27]. Disponível em: http://repositorio. ufba.br/ri/handle/ri/17650

16. Bustamante-Teixeira MT, Faerstein E, Latorre MR. Técnicas de análise de sobrevida. Cad Saúde Pública. 2002;18(3):579-94. doi: https://doi.org/10.1590/ S0102-311X2002000300003
17. Nasti G, Talamini R, Antinori A, et al. AIDS-related Kaposi's sarcoma: evaluation of potential new prognostic factors and assessment of the AIDS Clinical Trial Group Staging System in the Haart Era--the Italian Cooperative Group on AIDS and Tumors and the Italian Cohort of Patients Naive From Antiretrovirals. J Clin Oncol. 2003;21(15):2876-82. doi: https://doi.org/10.1200/ JCO.2003.10.162

18. El Amari EB, Toutous-Trellu L, Gayet-Ageron A, et al. Predicting the evolution of Kaposi sarcoma, in the highly active antiretroviral therapy era. AIDS. 2008;22(9):1019-28. doi: https://doi.org/10.1097/ QAD.0b013e3282fc9c03

19. Kasturia SE, Gunthel C, Zeng C, et al. Severe Kaposi sarcoma in an urban public hospital. AIDS Res Hum Retroviruses. 2017;33(6):583-9. doi: https://doi. org/10.1089/AID.2016.0141

20. Schwartz RA, Micali G, Nasca MR, et al. Kaposi Sarcoma: a continuing conundrum. J Am Acad Dermatol. 2008;59(2):179-206. doi: https://doi. org/10.1016/j.jaad.2008.05.001

Recebido em 6/7/2020 Aprovado em 14/8/2020 